\title{
Ist orales Ketamin eine Behandlungsoption?
}

Fragestellung: Vorstellung der aktuellen Datenlage einer oralen Darreichung von Ketamin bei therapierefraktären Depressionen und chronischen Schmerzen.

Hintergrund: Aktuelle Studien zur intravenösen Ketamin-Gabe bei schweren Depressionen zeigen vorteilhafte Kurzzeiteffekte. Im Rahmen der Behandlung von chronischen Schmerzsyndromen wird Ketamin schon länger eingesetzt und es sind auch schon verschiedene Darreichungsformen (oral, intravenös, intranasal, subkutan) besser untersucht.

Patienten und Methodik: Diese Übersichtsarbeit vergleicht verschiedene Darreichungsformen von Ketamin bei Patienten mit Depressionen und chronischen Schmerzsyndromen. Über eine PubMed-Suche wurden 88 entsprechende Artikel identifiziert und hinsichtlich der unter-

Schoevers RA, Chaves TV, Balukova SM et al. Oral ketamine for the treatment of pain and treatment-resistant depression. $\mathrm{Br} \mathrm{J}$ Psychiatry 2016; 208: 108-13 schiedlichen Dosierungsregime, der Patientenzahl, der Tage unter Ketamin, der Effekte, unerwünschter Arzneimittelwirkungen und der Studienqualität untersucht.
Ergebnisse: Insgesamt zeigte sich für die Behandlung von Depressionen eine nur niedrige methodische Stärke der evaluierten Studien, unabhängig von der Darreichungsform. Die verwendeten Dosierungen im Rahmen einer antidepressiven Therapie waren niedriger als bei analgetischer Therapie. Dieser Vergleich zwischen antidepressiver und analgetischer Dosierung wurde durch eine Umrechnung in eine Äquivalenzdosis zwischen der intravenösen und der weiteren Darreichungsformen umgesetzt. Hierbei zeigte sich, dass die Dosierungen bei Studien zu Depressionen deutlich im subanalgetischen Bereich lagen und die Medikationsdauer (in Tagen) kürzer war. Die Studienergebnisse zu der oralen Darreichungsform im Rahmen einer Schmerztherapie - zumindest übertragen auf die Behandlung einer Depression - lassen ein akzeptables Nebenwirkungsprofil annehmen.

Schlussfolgerungen: Eine orale Ketamin-Therapie erscheint, auch über einen längeren Zeitraum, eine nebenwirkungsarme und gut tolerierte Behandlungsalternative darzustellen. Allerdings liegen bislang nur sehr wenige systematische Untersuchungen vor, die auch mögliche Langzeitfolgen untersucht haben.

\section{- Kommentar von Joji Kuramatsu, Erlangen}

\section{Für eine Therapieempfehlung fehlen Langzeitdaten}

Diese Übersichtsarbeit unternimmt den Versuch, anhand der bislang zur Verfügung stehenden Daten die möglichen Implikationen einer oralen Ketamin-Therapie zur Behandlung von Depressionen zu analysieren. Allerdings konnte aufgrund der spärlichen Datenlage nur eine explorative Beschreibung dieses Therapieansatzes erfolgen, ohne das durch metaanalytische Verfahren eine tatsächliche Bewertung dieser Therapie möglich war. Explizit lagen zu dem Zeitpunkt der Veröffentlichung der vorliegenden Arbeit nur vier Studien beziehungsweise Fallberichte mit insgesamt 22 Patienten vor. Die Studie mit der größten Fallzahl, die hier Erwähnung findet, hat 14 Patienten mit oralem Ketamin (0,5 mg/kg/Tag) über 28 Tage behandelt [1]. Es zeigte sich - zwar ohne randomisiertes Studiendesign - eine über den Studienzeitraum dauerhaft signifikante Verbesserung der auf konventionellen Skalen gemessenen Werte im Vergleich zum Ausgangswert für Angst und Depression nach drei beziehungsweise 14 Tagen [1]. Hier ist zu erwähnen, dass eine aktuelle Studie aus dem November 2016 die orale Ketamin-Therapie bei Depressionen mit einem doppelblinden, randomisierten Studiendesign untersucht hat [2]. In dieser Arbeit wurden $50 \mathrm{mg}$ Ketamin pro Tag mit 150 mg Diclofenc täglich über einen Zeitraum von sechs Wochen verglichen. Es zeigten sich auch in dieser Arbeit, mit dem bislang validesten Studiendesign und der größten Patientenanzahl $(n=40)$, signifikante Unterschiede zugunsten einer oralen Ketamin-Therapie bei Patienten mit Depression und chronischen Schmerzen [2]. Insgesamt erscheint die orale Ketamin-Therapie ein vielversprechender Ansatz zur Behandlung schwer depressiver Patienten zu sein, allerdings bleibt ein ganz wesentlicher Aspekt unklar. Diesen Punkt heben ebenfalls die Autoren der Übersichtsarbeit deutlich hervor: Es fehlen bislang Daten zur Langzeitsicherheit einer Ketamin-Therapie und ebenso besteht ein theoretisch verändertes Risiko eines Missbrauchs durch Ketamin in Tablettenform. Somit ist für diese Therapieform nun in Anbetracht dieser aktuellen Arbeit zwar ein moderates Evidenzniveau vorhanden, jedoch sind weitere, deutlich größere Studien mit einem langfristigen Beobachtungsverlauf unabdingbar, um in einer praxisnahen Therapieempfehlung zu münden.

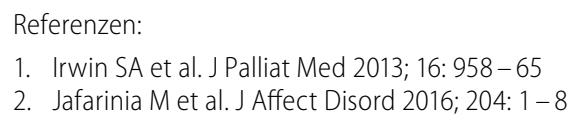

Dr. med. Joji B. Kuramatsu, Erlangen

Neurologische Klinik,

Universitätsklinikum Erlangen

E-Mail: joji.kuramatsu@uk-erlangen.de 